\title{
Fatores de Risco para Natimortalidade em um Hospital Universitário da Região Sul do Brasil
}

\author{
Risk Factors for Stillbirth at a Universitary Hospital in Southern Brazil
}

Kátia Vardanega, Dino Roberto Soares De Lorenzi,

Wilson Paloshi Spiandorello, Maíra Fernandez Zapparoli

\begin{abstract}
RESUMO
Objetivo: estudar a influência da assistência pré-natal e de fatores materno-fetais nas taxas de natimortalidade de um hospital universitário da região sul do Brasil.

Métodos: estudo caso-controle de 61 casos de natimortos ocorridos antes do início do trabalho de parto no Hospital Geral da Universidade de Caxias do Sul, RS, entre março de 1998 e junho de 2001. Os controles ( $n=224)$ foram selecionados aleatoriamente entre os fetos nascidos vivos no mesmo período. A análise da qualidade da atenção pré-natal baseou-se nos critérios estabelecidos pelo Programa de Humanização do Parto e Nascimento do Ministério da Saúde (PNHPN, 2000). Para avaliar possíveis fatores de risco de natimortalidade foi utilizado o odds ratio (OR). Eventuais fatores de confusão foram avaliados por meio de regressão logística.

Resultados: observou-se maior prevalência de prematuridade entre os casos de natimortos (idade gestacional média de $31,7 \pm 4,7$ vs $38,6 \pm 0,9$ semanas). O peso médio entre os natimortos foi de $1.705 \mathrm{~g}( \pm 837 \mathrm{~g})$ e de $3.080 \mathrm{~g}( \pm 576 \mathrm{~g})$ entre os controles. Acompanhamento pré-natal foi referido por $81,5 \%$ das mães dos natimortos e $91,6 \%$ do grupo controle. A análise inicial revelou associação de três fatores com o evento da natimortalidade: a atenção pré-natal inadequada quanto ao número de consultas médicas e solicitação de exames complementares básicos (43,6 vs 23,4\%), a história prévia de natimortalidade (6,6 vs $0,9 \%$ ) e a idade materna (27士7,9 anos vs $24 \pm 6,4$ anos). Todavia, após o ajustamento desses resultados pela regressão logística, apenas a idade materna manteve associação significativa com o óbito fetal. Conclusões: no presente estudo, a idade materna foi o fator mais fortemente associado com a natimortalidade.
\end{abstract}

PALAVRAS-CHAVE: Natimorto. Óbito fetal. Pré-natal.

\section{Introdução}

A mortalidade perinatal tem sido um dos indicadores mais utilizados quando o objetivo é avaliar as condições de saúde de uma população, visto que seu estudo revela informações valiosas acerca da qualidade da assistência prestada à mulher durante o ciclo gravídico-puerperal ${ }^{1-3}$.

$\mathrm{O}$ crescente interesse atualmente observa-

Serviço de Obstetrícia do Hospital Geral da Universidade de Caxias do Sul.

Correspondência:

Dino Roberto Soares De Lorenzi

Rua Bento Gonçalves 1759 / 602

95020-412 - Caxias do Sul - RS

e-mail: dlorenzi@zaz.com.br do em relação à mortalidade perinatal está relacionado ao fato de que, apesar de se verificar tendência mundial de declínio das taxas de mortalidade infantil, em particular na América Latina, as causas ligadas à gestação e ao parto contribuíram com somente $6 \%$ para a redução total dos óbitos infantis, passando a ocupar o primeiro lugar entre as causas de óbito de crianças no seu primeiro ano de vida ${ }^{4,5}$.

Particularmente, a redução das taxas de mortalidade perinatal, verificada nos países desenvolvidos a partir da segunda metade do século 20 , deveu-se principalmente à diminuição do seu componente neonatal precoce e não dos óbitos fetais, cujo declínio foi comparativamente menor, fazendo com que estes se tornassem a principal causa 
de morte perinatal nesses países. Esta tendência é atribuída aos contínuos progressos observados nos campos da Obstetrícia, pela melhoria da assistência pré-natal, e da Neonatologia, esta última principalmente através da criação dos centros de terapia intensiva neonatal, o que tornou possivel uma maior sobrevida de fetos nascidos pré-termo ${ }^{4-6}$.

Além disso, ao contrário dos países desenvolvidos, onde as perdas perinatais estão relacionadas principalmente com causas de difícil prevenção, entre elas as malformações congênitas, nos países subdesenvolvidos, como o Brasil, entre as causas de óbito perinatal destacam-se as afecções passivveis de prevenção ou controle por meio de adequada assistência pré-natal e ao parto, entre estas as síndromes hipertensivas, a sifilis congênita, o tocotraumatismo e a asfixia perinatal $^{5-8}$.

Assim, vários autores têm destacado a importância da natimortalidade como indicador de saúde perinatal ${ }^{3,6}$. A morte fetal, a despeito das conquistas tecnológicas observadas atualmente na área médica, não é entidade rara, acometendo ainda de 0,6 a $1,2 \%$ das gestantes na segunda metade da gestação. Entretanto, apesar de o estudo da natimortalidade revelar importantes informações a respeito da qualidade da assistência perinatal, esta ainda tem sido objeto de pouca atenção, sendo raramente estudada separadamente da mortalidade perinatal geral ${ }^{3}$.

Entre os fatores que dificultam a utilização da natimortalidade como indicador de saúde perinatal, destaca-se a escassez de registros hospitalares fidedignos a respeito dos fetos natimortos, dificultando desse modo a realização de estudos que visem conhecer os fatores determinantes do óbito fetal e a possibilidade de sua prevenção ${ }^{6}$.

Portanto, em face da relevância do tema, em particular no campo da saúde pública, os autores propõem-se a estudar a influência da assistência pré-natal no evento da natimortalidade e a sua relação com fatores maternos e fetais.

\section{Pacientes e Métodos}

Trata-se de estudo caso-controle, retrospectivo, de 61 casos de natimortos ocorridos no Serviço de Obstetrícia do Hospital Geral da Universidade de Caxias do Sul (HG-UCS) no período de março de 1998 a junho de 2001. Os controles $(n=224)$ foram selecionados aleatoriamente entre os fetos nascidos vivos no mesmo período, na proporção de quatro controles para cada caso. $\mathrm{O}$ referido hospital é instituição pública, gerenciada pela Fundação Universidade de Caxias do Sul, sendo responsável pelo atendimento de cerca de 1800 partos/ano.

Definiu-se como natimorto todo feto com idade gestacional igual ou superior a 22 semanas e/ ou peso maior ou igual a 500 gramas cujo óbito ocorreu antes da separação completa do corpo materno e que, ao nascer, não tenha apresentado quaisquer das seguintes manifestações compatíveis com a vida: batimentos cardíacos, pulsação de cordão umbilical ou movimento de músculos voluntários, de acordo com a $10^{\mathrm{a}}$ Revisão da Classificação Internacional de Doenças (OMS, 1993) ${ }^{9}$.

Visando evitar possiveis vieses de análise decorrentes da interferência da condução do trabalho de parto por parte da equipe responsável pela assistência obstétrica, foram incluídos no presente estudo somente os casos de óbito fetal cujas mães ao chegarem ao hospital não se encontravam em trabalho de parto. Pelo mesmo motivo, foram excluídos também os fetos cujo óbito ocorreu após a internação hospitalar.

A avaliação da qualidade da atenção pré-natal baseou-se nos critérios estabelecidos pelo Programa de Humanização do Pré-natal e Nascimento do Ministério da Saúde (2000) no que se refere ao calendário de consultas médicas e solicitação de exames complementares: início do acompanhamento pré-natal obrigatoriamente até a $14^{\mathrm{a}}$ semana de gravidez; número mínimo de 6 consultas de pré-natal, com a seguinte distribuição durante a gestação: uma consulta no primeiro trimestre, duas no segundo e três no terceiro trimestre; solicitação de exames complementares considerados obrigatórios: hemograma, tipagem sangüínea e determinação do fator Rh materno, exame comum de urina, VDRL ( $1^{\mathrm{a}}$ consulta e após a $30^{\mathrm{a}}$ semana de gravidez, devendo ser novamente repetido por ocasião do parto), glicemia de jejum, teste anti-HIV (na $1^{\text {a }}$ consulta) e, quando indicado, teste de Coombs indireto (na primeira consulta e mensalmente a partir da $24^{\mathrm{a}}$ semana de gravidez) ${ }^{10}$.

Os dados estudados foram obtidos a partir da revisão dos prontuários médicos registrados no Serviço de Arquivo Médico (SAME) do Hospital Geral da Universidade de Caxias do Sul, sendo posteriormente digitados e submetidos à análise eletrônica através dos programas EPI INFO 6.0 e SPSS 2000. Aceitou-se um erro de tipo I (a) igual a 5\% e um erro do tipo II (b) de 20\%, para um intervalo de confiança de 95\%. Para avaliar a relação de risco da assistência pré-natal com o evento da natimortalidade foi utilizado o odds ratio (OR). Outros fatores intervenientes ou de confusão foram avaliados através de regressão logística. Por fim, somente foram descritas as análises que se mostraram estatisticamente significativas.

A renda familiar foi classificada de acordo 
com o somatório total dos rendimentos dos componentes do grupo familiar dividido pelo número de membros da família (renda per capita), tomandose como parâmetro referencial o salário mínimo em reais vigente na época de coleta dos dados. Já a escolaridade materna foi avaliada conforme a classificação do Ministério da Educação: primeiro grau ( $1^{\mathrm{a}}$ a $8^{\mathrm{a}}$ série), segundo grau ( $1^{\mathrm{a}}$ a $3^{\mathrm{a}}$ série) e superior (nivel universitário).

Por ser pesquisa que envolve seres humanos, esta foi previamente submetida à avaliação da Comissão de Ensino e Pesquisa do Hospital Geral/Universidade de Caxias do Sul, tendo sido aprovada.

\section{Resultados}

A idade materna média entre os casos foi de 27 anos ( $\pm 7,9$ anos). A idade máxima foi 46 anos e a mínima de 15 anos. No grupo controle, a média etária materna foi de 24 anos ( $₫ 6,4$ anos), sendo que a idade máxima foi 43 anos e a mínima 14 anos, tendo-se observado diferença significativa entre os dois grupos $(p=0,0001)$. Já em relação à renda familiar, estado marital e escolaridade, não se evidenciou diferença quando se compararam o grupo de mães com natimortos e os controles.

A paridade materna média entre os casos foi de 1,9 gestações $(\mathrm{DP}= \pm 1,9)$, valor este superior às 1,3 gestações $(\mathrm{DP}= \pm 1,5)$ observadas entre os controles. Dez das mulheres incluídas entre os casos $(16,4 \%)$ e $24(10,7 \%)$ do grupo controle referiram história prévia de abortos. Não se observou diferença significativa entre os dois grupos. Todavia, em relação à história prévia de natimortalidade, $6,6 \%(n=4)$ das gestantes incluidas entre os casos relataram um ou mais natimortos anteriores, enquanto que no grupo controle constatou-se apenas $2(0,9 \%)$ relatos de mortes fetais prévias. Esta diferença mostrou-se significativa (OR 7,8/ IC $1,2-63,8 / \mathrm{p}=0,006178$ ).

A idade gestacional média dos conceptos natimortos foi de 31,7 semanas $( \pm 4,7$ semanas), sendo a idade gestacional mínima de 22 semanas e a máxima de 43 semanas. Ainda neste grupo, houve um predomínio de fetos pré-termo (83\%), ou seja, com menos de 37 semanas de gravidez. No grupo controle, a idade gestacional média foi de 38,6 semanas ( \pm 1 semana).

Quanto ao sexo fetal, 28 (45,9\%) dos natimortos eram do sexo feminino e $54,1 \%(n=33)$ do sexo masculino. No grupo controle, 55,8\% $(n=125)$ dos neonatos eram do sexo feminino e $44,2 \%(n=99)$ do sexo masculino. A média do peso fetal no grupo dos casos foi de $1.705 \mathrm{~g}( \pm 837 \mathrm{~g})$, sendo o peso mínimo de 418 g e o máximo de 6.052 g, ao passo que no grupo controle o peso fetal médio foi de 3.080g Ł576 g), sendo o mínimo 770 g e o máximo 4.570 g.

Em 34 dos prontuários médicos dos fetos natimortos $(56,8 \%)$ constatou-se a ocorrência de complicações clínicas durante a gravidez (Tabela 1). Quanto ao momento do óbito fetal, este foi prévio à internação hospitalar em $90,2 \%(n=55)$, ao passo que em $6,5 \%(n=4)$ dos casos este ocorreu após a hospitalização materna (excluídos dois casos cujo momento do óbito fetal não foi possível determinar com precisão). Em 34,4\% (n=21) foi solicitado o exame anatomopatológico fetal.

Tabela 1 - Complicações clínicas durante a gravidez entre os natimortos estudados.

Complicações

Síndrome hipertensiva*

Malformação fetal 24

Descolamento prematuro de placenta

Ameaça de abortamento $\quad 2$

Sífilis

Placenta prévia

Diabete gestacional

2

Infecção do trato urinário

* Neste grupo foram incluídas a hipertensão prévia à gestação e a doença hipertensiva específica da gestação.

** Hepatopatia, anemia, tiroidopatia e trabalho de parto pré-termo.

A avaliação dos grupos estudados quanto ao acompanhamento pré-natal revelou que, em $81,5 \%(n=44)$ dos natimortos estudados, as gestantes tiveram acompanhamento médico pré-natal, ao passo que no grupo controle esse percentual foi de $91,6 \%(n=153)$, não se obtendo diferença significativa entre os grupos no que se refere ao risco de óbito fetal. Todavia, visando contornar possiveis vieses ocorridos na análise anterior, procedeu-se à análise da atenção pré-natal entre os dois grupos estudados com base nos critérios de qualidade do Programa Nacional de Humanização do Parto e Nascimento do Ministério da Saúde. Assim, foi possivel constatar maior risco de ocorrência de natimortos entre as mulheres cujo pré-natal foi considerado inadequado (Tabela 2).

A média de consultas no pré-natal entre os casos foi de 3,8 consultas $(\underline{2}, 6)$, ao passo que no grupo controle, esta foi de 5 consultas $( \pm 2,6)$. Ao se compararem os casos cujo pré-natal foi considerado inadequado com a situação de não-realização de pré-natal não se evidenciaram diferenças significativas quanto ao risco de natimortalidade. 
Tabela 2 - Distribuição da população estudada quanto à adequação da atenção prénatal segundo os critérios do Programa de Humanização do Parto e Nascimento (Ministério da Saúde, 2000)

\begin{tabular}{lcc}
\hline Atenção pré-natal & Controles & Casos \\
& $\mathbf{n}(\%)$ & $\mathbf{n}(\%)$ \\
\hline Adequada & $111(76,6)$ & $22(56,4)$ \\
Inadequada & $34(23,4)$ & $17(43,6)$ \\
Total & 145 & 39 \\
\hline
\end{tabular}

Excluídos os casos ignorados e os casos em que não houve acompanhamento prénatal

$\mathrm{OR}=2,52(\mathrm{IC} 1,1-5,7) \mathrm{p}=0,02$

As variáveis independentes que se associaram com a ocorrência de óbito fetal foram a assistência pré-natal considerada inadequada $(p=0,001)$, a história de natimortos prévios $(p=0,02)$ e a idade materna $(p=0,001)$. Após o ajustamento das variáveis maternas e da qualidade da assistência pré-natal pela regressão logística e análise multivariada, constatou-se que apenas a idade materna e a adequação do acompanhamento pré-natal mantiveram influência significativa sobre as taxas de natimortalidade $(\mathrm{p}=0,001$ e $\mathrm{p}=0,002$, respectivamente).

Esse resultado se manteve quando as variáveis foram analisadas conjuntamente, porém, quando se inseriu uma nova variável que foi a interação entre estas duas, observou-se que somente a idade materna manteve significância estatística (OR 2,9/IC 1,2-3,8/p=0,0002). O aumento da idade materna associou-se com um aumento da incidência de óbitos fetais.

\section{Discussão}

É reconhecido que a análise das características sociais, demográficas e biológicas das gestações cuja resolução foi o óbito fetal fornece informações valiosas para a formulação de políticas de saúde que visem a redução da mortalidade perinatal, uma vez que permite a identificação de possiveis fatores de risco para sua ocorrência e, conseqüentemente, a sua prevenção ou controle c,11,12. $^{6}$.

Para Backett et al. ${ }^{13}$, a mensuração dos fatores de risco perinatais exige o conhecimento da história natural das complicações da gravidez e do parto, assim como um estudo epidemiológico de cada ocorrência definida em relação ao ambiente local. A prevalência de fatores de risco em diferentes comunidades e a possibilidade de intervenção em cada situação determinará as prioridades a serem estabelecidas.

Vários autores têm salientado que a aten- ção pré-natal pode consistir por si só fator de risco importante para óbito fetal ${ }^{6,14}$. Em seus estudos, Laurenti e Buchalla ${ }^{15}$ demonstraram que o risco gestacional era menor entre gestantes que se submeteram a sete ou mais consultas de pré-natal, visto que estas apresentavam menores taxas de mortalidade perinatal. Achado semelhante foi obtido por Rouquayrol et al. ${ }^{16}$, ao investigar fatores de risco para natimortalidade na cidade de Fortaleza. Estes autores constaram que a ausência de acompanhamento médico pré-natal ou o número insuficiente de consultas estava fortemente associado à ocorrência de morte fetal ${ }^{16}$.

Hoje é reconhecido que a assistência prénatal inclui metas extremamente amplas. Além de suas finalidades psicológicas, educacionais e sociais, visa prioritariamente a identificação das gestações potencialmente de risco, cuja condução requer manejo individualizado e especializado. A identificação dos casos de maior risco gestacional permite o seu encaminhamento precoce aos centros de atenção terciária, inclusive a hospitalização quando esta for indicada, o que permite a redução da mortalidade tanto perinatal quanto materna ${ }^{6,8,17}$.

Neste contexto, ganha dimensão a proposta atual de se incrementar o uso da natimortalidade como indicador de saúde, visto que a maioria dos estudos que abordam a questão do risco perinatal concentra-se nos achados de neomortalidade. A natimortalidade, apesar de ainda pouco explorada pelas estatísticas oficiais, fornece informações valiosas sobre as condições de assistência perinatal, tornando-a assim evento sentinela da qualidade da atenção pré-natal e ao parto ${ }^{15}$.

A própria identificação das causas de óbito fetal é por si só extremamente importante. A constatação do predomínio de doenças passiveis de prevenção ou controle por meio do seu diagnóstico precoce durante a gestação, tais como a sífilis congênita ou as síndromes hipertensivas, permite questionar a qualidade da atenção pré-natal. Já o predomínio de perdas fetais associadas a tocotraumatismo ou anóxia fetal intraparto leva ao questionamento da assistência ao parto ${ }^{3,18}$.

Apesar de no presente estudo não terem sido verificados diferenças significativas no que se refere à paridade materna, esta tem sido destacada na literatura como fator relacionado com o resultado perinatal ${ }^{6}$. Trevisan et al. ${ }^{19}$, ao estudar a atenção pré-natal no município de Caxias do Sul, constataram que quanto maior o número prévio de filhos, mais tardio era o início do acompanhamento pré-natal, podendo assim comprometer o resultado perinatal. De modo similar, De Lorenzi et al. ${ }^{18}$ demonstraram uma maior prevalência de óbitos fetais entre gestantes com maior paridade. 
A constatação entre os conceptos natimortos estudados de que o peso médio ao nascer era inferior ao observado no grupo controle (1.705 g vs 3.080 g), é explicável pela maior taxa de prematuridade observada entre os casos de óbito fetal $(31,7$ vs 38,6 semanas). Laurenti e Buchalla ${ }^{20}$, ao estudar 12.999 nascimentos ocorridos em diferentes maternidades brasileiras, observaram que dos óbitos perinatais identificados, $60 \%$ eram de fetos com menos de $2.500 \mathrm{~g}$. De forma semelhante, Petridou $^{11}$, ao revisar 2006 casos de natimortalidade ocorridos na Grécia entre 1989 e 1991, verificou percentual de 59,4\% de óbitos intra-útero entre conceptos com peso inferior a $2.500 \mathrm{~g}$.

Em relação à idade materna, Faúndes et al. ${ }^{21}$ destacaram que a faixa etária de menor risco obstétrico estava entre 20 e 24 anos, aumentando nos extremos da vida reprodutiva, em particular, após os 35 anos de idade. Todavia, entre as adolescentes, os mesmos autores verificaram percentual menor de complicações perinatais, o que talvez possa ser explicado pela tendência atual de implementação de programas de saúde voltados para o atendimento de adolescentes.

A constatação neste estudo de que a idade materna apresentava associação significativa com o evento da natimortalidade reforça os achados da literatura. Laurenti e Buchalla ${ }^{20}$ demonstraram que o coeficiente de natimortalidade era menor entre mães mais jovens, aumentando progressivamente a partir dos 35 anos, até atingir o seu valor máximo após os 45 anos. Esses resultados foram similares aos encontrados por Little e Weinberg $^{17}$ que, ao relacionarem a idade materna e a incidência de natimortalidade, constataram maior risco relativo entre mulheres com mais de 35 anos $(R R=1,61)$. Entre as explicações para o maior risco de óbito fetal entre gestantes com idade superior a 35 anos está a maior prevalência de anomalias congênitas entre essas mulheres. Estudos evidenciaram que a incidência de malformações até os 34 anos é de cerca de 0,75\%, percentual este que aos 35 anos chega a 1,29\%, aumentando progressivamente a cada ano de vida da mulher ${ }^{22}$.

Paralelamente, foi demonstrada também a maior prevalência de doenças crônico-degenerativas e de afecções específicas da gravidez entre mulheres com idade superior a 35 anos, o que promove aumento do seu risco gestacional ${ }^{6,18}$. Ziadeh e Yahaya $^{23}$. evidenciaram predominio de complicações obstétricas entre gestantes com idade maior ou igual a 40 anos, o que também foi observado por Seoud et $\mathrm{al}^{24}$. Estes autores constataram maior risco de prematuridade, restrição de crescimento intra-uterino e complicações clínicas maternas, como síndromes hipertensivas e diabete, entre mulheres com mais de 40 anos e, principalmente, entre multíparas.

Não obstante tais achados justificarem os resultados obtidos pelo presente estudo, é necessário tecer algumas considerações acerca da influência da atenção pré-natal nas taxas de natimortalidade da população pesquisada. Talvez, o fato de ser estudo retrospectivo tenha interferido nos achados verificados. De Lorenzi et al. ${ }^{18}$, ao pesquisarem as causas de natimortalidade entre usuárias do Sistema Único de Saúde de Caxias do Sul, observaram que o preenchimento dos prontuários hospitalares nos casos de óbito fetal era freqüentemente incompleto e até vago, principalmente por parte dos profissionais médicos, o que pode ter se refletido nos dados coletados.

Além disso, a análise da qualidade da atenção pré-natal com base somente no número de consultas realizadas e solicitação de todos os exames complementares, conforme as recomendações do Programa de Humanização do Parto e Nascimento do Ministério da Saúde $(2000)^{10}$, pode ter sido insuficiente, visto que não contempla as questões de educação em saúde e preparo para o parto, hoje reconhecidamente imprescindiveis no acompanhamento pré-natal. As mulheres devidamente orientadas e conscientes dos cuidados necessários durante a gestação contribuem para que esta transcorra em segurança significativamente maior. Inúmeros trabalhos têm destacado a importância de que não somente o número mínimo de consultas de pré-natal seja atingido, mas que cada atendimento seja também resolutivo, quando o objetivo é preservar a saúde tanto materna quanto fetal ${ }^{17,19}$.

Portanto, apesar de este estudo ser válido por destacar a importância atual das taxas de natimortalidade como indicador de saúde perinatal, cujo estudo pelo método epidemiológico é de baixo custo e fácil execução, os autores destacam a necessidade da coleta prospectiva de dados, a fim de evitar possíveis vieses de aferição.

\section{ABSTRACT}

Purpose: to study the influence of the prenatal care, fetal and maternal factors on the stillbirth rates of a university hospital from the south of Brazil.

Methods: a case-control study of the cases of stillbirth occurred before the beginning of labor, from March 1998 to June 2001, at the Hospital Geral of Caxias do Sul University. The controls were selected among live newborns. The analysis of the quality of the prenatal care was based on the criteria established by the Programa de Humanização do Pré-natal e 
Nascimento of the Brazilian Health Ministry (2000). To evaluate possible risk factors for stillbirth we used the odds ratio (OR). Other confounding factors were evaluated by logistic regression.

Results: preterm delivery was more prevalent in the cases of stillbirth (31.7+4.7 vs 38.6+0.9). The average birth weight among the stillborns was $1,705 \mathrm{~g}( \pm 837 \mathrm{~g})$, while in the controls it was 3,080 $\mathrm{g}( \pm 576 \mathrm{~g})$. Prenatal care was observed in $81.5 \%$ of the stillbirths and in $91.6 \%$ of the control group. The initial analysis showed that three factors were associated with stillbirth: inadequate prenatal care (43.6 vs $23.4 \%$ ), history of previous stillbirth (6.6 vs $0.9 \%$ ) and maternal age $(27 \pm 7.9$ years vs $24 \pm 6.4$ years $)$. Nevertheless, after adjustment of these variables through logistic regression, only the maternal age maintained its association with the stillbirth rates.

Conclusions: in the present study, the strongest factor associated with the occurrence of stillbirth was the increase in maternal age.

KEY WORDS: Stillbirth. Fetal death. Prenatal care.

\section{Referências}

1. Barros FC, Victora CJ, Vaughan JP. Causas de mortalidade perinatal em Pelotas, RS (Brasil): utilização de uma classificação simplificada. Rev Saúde Pública 1987; 21:310-6.

2. Centurión CAS. Avaliação da qualidade de assistência pré-natal no município de Taboão da Serra [tese]. São Paulo: Universidade de São Paulo; 1994.

3. Aquino MM, Cecatti JG. Epidemiologia do óbito fetal em população de baixa renda. Rev Bras Ginecol Obstet 1998; 20:71-5.

4. Laurenti R, Buchalla CM, Costa Jr ML. Estudo da morbidade e da mortalidade perinatal em maternidades: I - Descrição do projeto e resultados gerais. Rev Saúde Pública 1984; 18:436-7.

5. Nogueira M. Assistência pré-natal: prática de saúde a serviço da vida. $1^{\text {a }}$ ed. São Paulo: Hucitec/Abrasco; 1994.

6. De Lorenzi DRS, Tanaka ACA, Bozzetti MC, Ribas FE, Weissheimer L. A natimortalidade como indicador de saúde perinatal. Cad Saúde Pública 2001; 17:141-6.

7. Tanaka ACA. Estatísticas de mortalidade perinatal. In: Anais do Simpósio Franco-Brasileiro de Metodologias de Pesquisa em Saúde Perinatal; dia mês? 1994; São Paulo, Brasil. São Paulo: Faculdade de Saúde Pública/USP; 1994. p.13-20.

8. Aerts DRGC. Investigação dos óbitos perinatais e infantis: seu uso no planejamento de políticas públicas de saúde. J Pediatr 1997; 73:364-6.

9. Organização Mundial da Saúde. Classificação estatística internacional de doenças e problemas relacionados à saúde. 10 $0^{\underline{a}}$ Revisão. São Paulo: Edusp; 1993.
10.Brasil. Ministério da Saúde. Secretaria de Políticas de Saúde. Programa de humanização do pré-natal e nascimento. Brasília; 2000.

11.Petridou E, Kotsifakis G, Revinthi K, Polychronopoulou A, Trichopoulos D. Determinants of stillbirth mortality in Greece. Soz Präventivmed 1996; 41:70-8.

12.Lammer EJ, Brown LE, Anderka MT, Guyer B. Classification and analysis of fetal deaths in Massachussetts. JAMA 1989; 261:1757-62.

13. Backett EM, Davies AM, Petros-Barviazan A. El concepto de riesgo en la asistencia sanitaria: con especial referencia la salud materno-infantil y a la planificación familiar. Ginebra: Organización Mundial de la Salud; 1984.

14.Greenwood R, Golding J, McCaw-Binns A, Keeling J, Ashley D. The epidemiology of perinatal death in Jamaica. Paediatr Perinat Epidemiol 1994; 8 (Suppl 1): 143-57.

15.Laurenti R, Buchalla CM. Indicadores da saúde materna e infantil: implicações da décima revisão da Classificação Internacional de Doenças. Rev Panam Salud Publica 1997; 1:18-22.

16. Rouquayrol MZ, Correia LL, Barbosa LMM, Xavier LGM, Oliveira JW, Fonseca W. Fatores de risco de natimortalidade em Fortaleza: um estudo de casocontrole. J Pediatr 1996; 72:374-8.

17.Little RE, Weinberg CR. Risk factors for antepartum and intrapartum stillbirth. Am J Epidemiol 1993; 137:1177-89.

18.De Lorenzi DRS, Tanaka ACA, Ribas FE, Rech FS, Weissheimer L. Perfil epidemiológico da natimortalidade em Caxias do Sul - RS. Rev Cient AMECS 1999; 8:29-33.

19.Trevisan MR, De Lorenzi DRS, Araújo NM, Esber K. Perfil da assistência pré-natal entre usuárias do Sistema único de Saúde em Caxias do Sul. Rev Bras Ginecol Obstet 2002; 24:293-9.

20.Laurenti R, Buchalla CM. Estudo da morbidade e mortalidade perinatal em maternidades: II Mortalidades perinatal segundo o peso ao nascer, idade materna, assistência pré-natal e hábito de fumar da mãe. Rev Saúde Pública 1985; 19:225-32.

21.Faúndes A, Fanjul B, Henriquez G, Mora G, Tognola C. Influencia de la edade y de la paridad sobre algunos parametros de morbilidad materna y sobre la mortalidad fetal. Rev Chil Obstet Ginecol 1972; 37:6-14.

22.Magalhães JA, Lemos NA, Cavalheiro J. Retardo do crescimento intra-uterino. In: Freitas F, MartinsCosta SH, Ramos JGL, Magalhães A, organizadores. Rotinas em Obstetrícia. 3 $3^{\text {a }}$ ed. Porto Alegre: Artes Médicas; 1997. p.74-83.

23.Ziadeh S, Yahaya A. Pregnancy outcome at age 40 and older. Arch Gynecol Obstet 2001; 265:30-3.

24.Seoud MA, Nassar AH, Usta IM, Melhem Z, Kazma A, Khalil AM. Impact of advanced maternal age on pregnancy outcome. Am J Perinatol 2002; 19:1-8.

Recebido em: 30/8/2002 Aceito com modificações em: 31/10/2002 